\title{
ASSESSMENT OF LEVEL OF TOXIC HEAVY METAL (PB) IN LOCAL AND FOREIGN BRANDS OF LIPSTICKS IN FCT, ABUJA, NIGERIA
}

\author{
Ayuba Samali ${ }^{1}$, ${ }^{*}$ Halima Zubairu Lawal ${ }^{1}$, and Gevevieve, Ofozor ${ }^{2}$ \\ ${ }^{1}$ Department of Medicinal Chemistry and Quality Control (MC \& QC), National Institute for \\ Pharmaceutical Research and Development (NIPRD), Federal Ministry of Health, Idu Industrial Area, \\ Abuja Nigeria \\ ${ }^{2}$ Department of Biochemistry, Federal University of Technology Minna, Niger State, Nigeria. \\ *Corresponding author: yarlawal@gmail.com 08036928415
}

\begin{abstract}
This study determined toxic heavy metal concentration in Local and Foreign brands of lipsticks sold in FCT, Abuja Nigeria. The study analyzed twenty (20) samples of lipsticks comprising of ten (10) Local and ten (10) Foreign brands for Lead using flame atomic absorption spectrophotometric (FAAS) method. The mean concentration range of Lead in both the Local and the Foreign lipstick samples and their estimated daily intake by average users are 22-714.70 $\mu \mathrm{g} / \mathrm{g}$ and $74.9-47927 \mu \mathrm{g} / \mathrm{g}$ with the estimated daily intake by the average users as 1.056- 34.305 $\mu \mathrm{g} / \mathrm{g}$ (Local samples) and $3.595-2300.496 \mu \mathrm{g} / \mathrm{g}$. (Foreign), while the estimated daily intake by high users are $3.828-124.358 \mu \mathrm{g} / \mathrm{g}$ (Local) and $13.023-8339.298 \mu \mathrm{g} / \mathrm{g}$ (Foreign). The estimated concentration of Lead ingested daily intake in the Local and the Foreign samples indicated 20\% and $70 \%$ of the samples were not safe for the average users. While the ingested daily intake in the Local and the Foreign samples indicated $50 \%$ and $90 \%$ of the samples were also not safe for high users as compared to WHO limit $(20 \mu \mathrm{g} / \mathrm{g})$ for Lead specified in cosmetic. Thus, continuous usage of these lipsticks can increase rate of exposure of the body system to Lead related disease conditions such as cancer which is known to be detrimental.

Keywords: Lipstick, Lead, AAS, Health hazard,
\end{abstract}

\section{INTRODUCTION}

Cosmetics have often been considered as more harmful by many dermatologists than good due to the complexity of the ingredients used in their formulation. Most of which are linked to many diseases conditions such as cancer, genetic aberration, developmental and reproductive problems (Gondal et al., 2010). Lipstick is one of the most commonly used cosmetic products which are produced from a range of chemicals. For most women, the use of lipstick is not only for fashion statement but as a necessity for their everyday outdoor activity. Survey of women on the use of lipstick shows that about $75 \%$ of women make use of varying brands of lipsticks (Zhao et al., 2013), it was also reported that about $90 \%$ of lipstick users cannot leave their homes without the application of lipstick, in other words, "it is a must for most of these women". However, the ingestion of these lipsticks on a daily base gives one a course of concern as it would be important to be aware of what is ingestible in these lipsticks. Generally, waxes and oils make the bulk of lipstick composition; a single stick of lipstick can contain several different chemical compounds with few substances and compounds whose inclusion is essential. This involves careful choice of the ingredients in order to obtain the desired colour, glossiness, and indelibility (Iman and Sami, 2011). In recent years there has been concern over the very small amounts of heavy metals that can be found in some lipsticks. A recent study of 32 popular lipsticks found trace contaminant amounts of lead, cadmium, aluminum, chromium and manganese. The slow release of these metals into human system may be harmful to biological system if allowed to accumulate over time. The metals could accumulate in the body organs due to their long half life and interfere with essential nutrients of similar oxidation states such as calcium and zinc (Adepoju-Bello and Alabi, 2012). For instance, the toxicity of lead at high concentrations of exposure is well documented but a major concern in recent time is the possibility that continual exposure to even relatively low levels of this toxic metal in lipsticks products may pose potential health risks such as impaired renal, hemopoietin and nervous system with different reports linking to loss of cognitive function (Koller et al., 2004; Ullah et al., 2017). 
The presence of lead in cosmetics has also been reported and thus the European union for cosmetic banned lead and lead compounds in cosmetics since 1976 and strict adherence to quality control is essential in ensuring that lead contamination in cosmetic product is prevented (Amit et al, 2010).

The growing concern about the physiological and behavioral effects of toxic metals on human health and population cannot be overlooked. This study was initiated due to several commonly reported cases of lead related disease conditions in women which could possibly be linked with use of cosmetic products such as lipstick. The aim was to evaluate the Local and Foreign brands of lipsticks commonly sold in FCT, Abuja Nigeria for Lead content in order to ascertain their level of safety.

\section{MATERIALS AND METHODS \\ Materials:}

All the reagents used were of analar grades and purity. Porcelain crucibles, plastic sample bottles, appropriate glasswares used were properly washed using detergents and rinsed with deionised water. Other equipments used are water bath, Muffled furnace, Atomic Absorption Spectrometer and Analytical Plus.

\section{Sample Collection}

Twenty (20) brands of lipsticks which comprised ten (10) Local and ten (10) Foreign brands were purchased from different cosmetic stores in Federal Capital Territory (FCT), Abuja Nigeria. The samples were coded as LipL1, LipL2, LipL3, LipL4, LipL5, LipL6, LipL7, LipL8, LipL9 and LipL10 (Local brands) and LipF1, LipF2, LipF3, LipF4, LipF5, LipF6, LipF7, LipF8, LipF9 and LipF10 (Foreign brands) and were taken to the laboratory and kept at room temperature for subsequent preparation and analysis.

\section{Reagents and standards}

Analytical grade concentrated nitric acid (S.G. 1.4 at $\left.25^{\circ} \mathrm{C} ; 69 \% \mathrm{~W} / \mathrm{V}\right)$ and concentrated I. Concentration:

$$
\text { Conc. }(\mu g / g)=\frac{\text { Conc. } \mu \frac{g}{m l} \times \text { volume. }}{\text { weight }(g)}
$$

II. Concentration of average estimated daily intake $(\mu \mathrm{g} / \mathrm{g})$ :

$$
\text { Conc. }=\frac{\text { Conc. obtained } \times \text { daily usage }}{\text { weight sample }}
$$

hydrochloric acid (S.G. 1.18; 37\% W/V) were used for sample preparation and standards for the metal of interest of concentrations 0.5, 2.5, 4.5 $6.5,8.5$ and $10.5 \mu \mathrm{g} / \mathrm{cm}^{3}$ by diluting $0.05,0.25$, $0.45,0.65,0.85$ and $1.05 \mathrm{~cm}^{3}$ of the standard stock solution (1000ppm) with deionized water using appropriate sizes of precision pipette and volumetric flask.

\section{Sample Preparation}

The glassware and plastic containers were thoroughly washed, rinsed with tap water and then soaked in $5 \%$ nitric $\left(\mathrm{HNO}_{3}\right)$ solution for 24 hours, rinsed with deionized water and dried prior to use. $0.5 \mathrm{~g}$ of each of the samples was weighed in a porcelain crucible and dry ash in a muffled furnace by stepwise temperature increase up to $550^{\circ} \mathrm{C}$ until completely ash (Ayenimo et al., 2010). The ash samples were digested over steamed water-bath with $12 \mathrm{~cm}^{3}$ of $20 \% \mathrm{HCl}$, diluted with deionized water, filtered through Whatman filter paper and made-up to mark in $50 \mathrm{~cm}^{3}$ volumetric flasks, transferred to sample plastic bottles and kept at room temperature for subsequent analysis.

\section{Samples Analysis}

The evaluation of heavy metal content of lipstick is vital due to safety issues associated to it. Ullah et al.(2013) reported various available analytical methods use for analysis of heavy metals. In this study, Flame Atomic Absorption Spectrometric (Model: GBC Avanta, Versin 2.0) method was used for the determination of Lead. The instrument operating conditions are: wavelength $217 \mathrm{~nm}$, lamp current at $5 \mathrm{~mA}$, slit width at $0.5 \mathrm{~nm}$, measurement mode was by integration, calibration mode was linear least square through zero and the flame type was air-acetylene (2.2513.10). The readings of the standards, blank and the analyte concentrations and absorbance were rounded off from measurements in triplicate. The data obtained were processed by calculating the actual concentration in the samples analyzed using the relation: 
III. Relative Intake Indices (RII):

$$
R I I=\frac{\text { Estimated daily intake }}{\text { Acepted daily intake }}
$$

\section{RESULTS AND DISCUSSION}

Table 1 shows the mean concentration of Lead in the Local and the foreign lipsticks analyzed. The results of the study obtained indicated higher concentration of Lead in the Foreign lipsticks as compared to the Local. The mean concentration and range of Lead in the overall $(n=10)$ Local and the Foreign lipstick analyzed are: 207.66 $\mu \mathrm{g} / \mathrm{g}$ (range $22-714.70 \mu \mathrm{g} / \mathrm{g}$ ) with the highest concentration being obtained in sample 2 (LipL2) and $5465.23 \mu \mathrm{g} / \mathrm{g}$ (range 74.90 - 47927.00 $\mu \mathrm{g} / \mathrm{g}$ ) with the highest concentration in sample 5 (LipF5). The results obtained from this study indicated higher concentration of Lead in the samples analyzed compared to results obtained from similar study reported by Brandão et al.
(2012) where the highest concentration obtained was $73.1 \pm 5.2 \mu \mathrm{g} \mathrm{g}^{-1}$. The concentrations obtained in all the samples analyzed were above the maximum permissible limits recommended (10ppm) by USA/FDA for Lead as impurities in cosmetics (U.S/FDA, 2016). Therefore, since simple absorption of Lead can be achieved after a consumer licks her lips, the health of the users' of both these Local and the Foreign lipsticks are under threat. Cosmetics manufacturers should therefore take responsibility for averting potentially harmful levels of Lead in their finished products by adopting good manufacturing practices that can achieve lower levels of Lead.

Table I: Mean Concentration $(\mu \mathrm{g} / \mathrm{g})$ of Lead in Lipsticks

\begin{tabular}{lll}
\hline S/N & Local Samples (LipL) & Foreign samples (LipF) \\
\hline Lip1 & $674.86 \pm 0.04$ & $551.00 \pm 0.21$ \\
Lip2 & $714.70 \pm 0.02$ & $74.90 \pm 0.05$ \\
Lip 3 & $28.59 \pm 0.02$ & $1414.70 \pm 0.55$ \\
Lip 4 & $27.24 \pm 0.11$ & $1474.90 \pm 0 . .40$ \\
Lip 5 & $22.00 \pm 0.01$ & $47927.00 \pm 0.35$ \\
Lip 6 & $33.73 \pm 0.10$ & $423.11 \pm 0.11$ \\
Lip 7 & $25.85 \pm 0.12$ & $1084.45 \pm 0.12$ \\
Lip 8 & $174.80 \pm 0.32$ & $1340.55 \pm 0.32$ \\
Lip 9 & $181.40 \pm 0.22$ & $187.50 \pm 0.11$ \\
Lip 10 & $193.40 \pm 0.50$ & $174.20 \pm 1.11$ \\
Mean & 207.66 & 5465.23 \\
Range & $22.00-674.86$ & $74.90-47927.00$ \\
\hline
\end{tabular}

The prediction of the daily intake of Lead residue for average users based on the most realistic estimation of the residue levels in lipstick was also determined and the results presented in Table 2 which indicates the estimated daily intake (EDI) of the mean and the ranges of concentrations of Lead from both the Local (LipL) and the Foreign samples (LipF) by average users' as $9.9675 \mu \mathrm{g} / \mathrm{g}(1.056-34.306 \mu \mathrm{g} / \mathrm{g})$ and 262.3454 $\mu \mathrm{g} / \mathrm{g}(9-2300.496 \mu \mathrm{g} / \mathrm{g})$ respectively. The results obtained were above the maximum limits of estimated daily intake (EDI) of Lead
$(0.024 \mu \mathrm{g} /$ day $)$ specified for average users' by $\mathrm{NIOSH}$. (2003). Health status of the average users of both the Local and the Foreign brands of the lipsticks are exposed to danger of Lead associated disease conditions such as cancer (Zhao et al., 2013). The findings of this study have corresponded with studies from other parts of the world which also reported high level of Lead lipsticks and other cosmetics (Hepp et al. 2009; Al-Saleh et al. 2009; FDA 2011; AdepojuBello et al. 2012; Brandao et al. 2012; Gondal et al. 2010) 
Table 2: Mean Concentration of Estimated Daily Lead Intake for Average Users.

\begin{tabular}{llc}
\hline & Concentration $(\mu \mathrm{g} / \mathrm{g})$ & \\
\hline Samples' code & Local Samples $(\mathrm{LipL})$ & Foreign samples $(\mathrm{LipF})$ \\
\hline Lip1 & 32.393 & 26.448 \\
Lip2 & 34.306 & 3.595 \\
Lip 3 & 1.372 & 67.905 \\
Lip 4 & 1.308 & 70.795 \\
Lip 5 & 1.056 & 2300.496 \\
Lip 6 & 1.619 & 20.309 \\
Lip 7 & 1.241 & 52.198 \\
Lip 8 & 8.390 & 64.346 \\
Lip 9 & 8.707 & 9.000 \\
Lip 10 & 9.283 & 8.362 \\
Mean & 9.9675 & 262.3454 \\
Range & $1.056-34.306$ & $9-2300.496$ \\
\hline
\end{tabular}

The mean and range concentrations of for the higher users of the Local and the Foreign brands are $36.1322 \mu \mathrm{g} / \mathrm{g} \quad(3.828-124.358 \mu \mathrm{g} / \mathrm{g})$ and $2597.735 \mu \mathrm{g} / \mathrm{g} \quad(13.033-8339.298 \mu \mathrm{g} / \mathrm{g}) \quad$ as presented in Table 3. The results obtained were higher as compared to the maximum limits for estimated daily intake (EDI) which is $0.087 \mu \mathrm{g} /$ day (Zhao et al., 2013).

Table 3: Mean Concentration of Estimated Daily Lead Intake for Higher Users

\begin{tabular}{llc}
\hline & Concentration $(\mu \mathrm{g} / \mathrm{g})$ & \\
\hline Samples' code & Local Samples $(\mathrm{LipL})$ & Foreign samples $(\mathrm{LipF})$ \\
\hline Lip1 & 117.425 & 95.874 \\
Lip2 & 124.358 & 13.033 \\
Lip 3 & 4.975 & 246.157 \\
Lip 4 & 4.739 & 256.633 \\
Lip 5 & 3.828 & 8339.298 \\
Lip 6 & 5.869 & 73.621 \\
Lip 7 & 4.497 & 188.694 \\
Lip 8 & 30.415 & 233.256 \\
Lip 9 & 31.564 & 32.625 \\
Lip 10 & 33.652 & 30.311 \\
Mean & 36.1322 & 2597.735 \\
Average & $3.828-124.358$ & $13.033-8339.298$ \\
\hline
\end{tabular}

\section{CONCLUSION}

The results obtained shown that Local and Foreign brands of lipsticks use by most Nigerian women are highly contaminated. Lead is found to be beyond permissible limit in cosmetics. This shows that users of this cosmetic product are

\section{REFERENCES}

Adepoju-Bello, A.A., Oguntibeju, O.O., Adebisi, R.A., Okpala, N. and Coker, H.A.B. (2012): Evaluation of the concentration of toxic metals in cosmetic products in Nigeria. African J. Biotechnol, 11:1636016364.

Al-Saleh, I; Al-Enazi, S. and Shinwari, N. (2009). Assessment of lead in cosmetic products. Regul Toxicol Pharmacol 54:105-113. highly exposed to hazardous chemicals therefore their health is at risk of Lead related disease conditions such as cancer. The result of this finding will be published for accessibility by the public in order to use it as caution tool to lipstick users.

Amit, S. C., Rekha, B., Atul, K. S., Sharad, S.L., Dinesh, K.C., and Vinayak, S.T. (2010). Determination of Lead and Cadmium in cosmetic products J. Chem. Pharm. Res., 2(6):92-97

Ayenimo, J.G., Yusuf, A.M., Adekule, A.S. and Makinde, O.W. (2010). Heavy metal exposure from personal care product. Bull. Environ. Contam. Toxicol. $84,8-14$ 
Brandao, J.D.O., Okonkwo, O.J., Sehkula, M. and Raseleka, R.M. (2012): Concentrations of Lead in Cosmetics commonly used in South Africa. Toxicol Environ Chem, 94 (1):70- 77.

FDA (U.S. Food and Drug Administration).(2011). Listing of Color Additives Exempt from Certification. 21CFR73.

http://www.accessdata.fda.gov/scripts/cd $\mathrm{rh} / \mathrm{cfdocs} / \mathrm{cfcfr} /$ CFRSearch.cfm?CFRPart= 73

EshowFR=1\&subpartNode=21:1.0.1.1.26. $\underline{3}$ [accessed26 July 2012].

Gondal, M.A., Seddigi, Z.S., Nasr, M.M. and Gondal, B. (2010): Spectroscopic Detection of Health Hazardous Contaminants in Lipstick using Laser Induced Breakdown

Spectroscopy. J. Hazard Meter, (175):726-732.

Hepp, N.M; Mindak, W.R. and Cheng, J. (2009). Determination of total lead in lipstick:

Development and validation of a microwave-assisted digestion, inductively coupled plasma-mass

spectrometric method. J. Cosmet. Sci. 60:405-414.

Iman, A.S and Sami, A.E (2011). "Trace metals in lipsticks". Toxicological \& Environmental Chemistry. 93 (6): 1149-1165.

Koller, K., Brown, T., Spurgeon, A. and Levy, L. (2004), Recent Development in Low Level Exposure and Intellectual Impairment in Children, Environ. Health Perspect. Vol. 112(9), pp. 987 - 994
NIOSH (National Institute for Occupational Safety and Health. (2003). NIOSH Manual of Analytical Methods. http://www.cdc.gov/niosh/docs/2003154/method-m.html [accessed 23 May 2017]

Nnorom, I.C. (2011). Trace metals in cosmetic facial talcum powders marketed in Nigeria. Toxicological and Environmental Chemistry, 6 (93), 1135-1148(14)

Ullah, H., Noreen, S., Fozia, C., Rehman,A., Waseem, A., Zubair, A., Adnan, M. and Ahmad, I. (2013). Comparative study of heavy metals content in cosmetic products of different

countries marketed in Khyber Pakhtunkhwa, Pakistan. Arabian Journal of Chemistry (2017) 10, 10-18

U.S. Food and Drug Administration (2016), “FDA's Testing of Cosmetics for Arsenic, Cadmium, Chromium, Cobalt, Lead, Mercury, and Nickel Content, accessed" 2017 at13.25PM.

http://www.fda.gov/Cosmetics/ProductsIngredie nts/PotentialContaminants/ucm452836.h tm.

Zhao, D.I., Jie, L., Chao, L., Juhasz, A.L.., Scheckerl, K.G., Luo, J., Hong-Bo, L. and Lena,Q.M.. (2013). Lead Relative Bioavailability in lip products and their potential Health Risk to Women. Environ. Sci. Technol., 50 (11), pp 60366043 